\title{
EDITORIAL
}

\section{Paediatric asthma: where to go?}

\author{
Mariëlle W. Pijnenburg
}

$\triangle$ sthma is the leading chronic disease in children in the Western world, affecting 5-20\% of school-aged children in Europe [1]. Childhood asthma is a serious public health problem. First, it causes considerable morbidity with high frequencies of sleep disturbances, emergency visits, school absence, and limitations of physical activity, and healthcare utilisation [2]. As quoted by HEDLIN et al. [3] in this issue of European Respiratory Review, asthma is the fourth most common cause of disability-adjusted life years for children aged 10-14 yrs. Secondly, as asthma is associated with reduced lung function growth, and lung function at a young age is a determinant of lung function in adult life, optimal treatment is of major concern for long-term prognosis [4, 5].

Several efforts have been made to improve knowledge on childhood asthma in order to unravel pathogenesis, and improve treatment modalities and monitoring strategies, as reflected by almost 2,000 papers published on asthma and children in 2011 and 2012. In this issue, HEDLIN et al. [3], in an extensive overview, bring together all novel findings on paediatric asthma and delineate future directions.

The GA ${ }^{2} L E N$ initiative is an inspiring example of how international collaboration may take a clinical problem, such as problematic severe asthma, further. The proposed work-up of asthmatic children who are uncontrolled on high doses of medication and the classification into "difficult to treat" or "therapy resistant" provides the clinician with practical tools on how to treat these patients.

However, several questions remain to be answered. As pointed out by HEDLIN et al. [3], heterogeneity of asthma in general and problematic asthma in particular makes it "problematic" to define phenotypes that provide useful information on pathophysiology, treatment, monitoring of disease and future risk. More objective methods are needed for phenotyping, such as cluster analysis or principle component analysis. It is yet to be determined whether noninvasive biomarkers in exhaled air or exhaled breath condensate and imaging techniques are useful in phenotyping, and whether they may lead to a more personalised treatment.

Remodelling, as briefly discussed by HeduIN et al. [3], is a poorly understood phenomenon, with unclear relationships with inflammation. It remains to be clarified whether remodelling is preventable and thus loss of lung function could be stopped.

CORRESPONDENCE: M.W. Pijnenburg, Dept of Paediatrics/Paediatric Respiratory Medicine Erasmus MC - Sophia Children's Hospital, Dr Molewaterplein 60, 3015 GJ Rotterdam, The Netherlands. E-mail: m.pijnenburg@erasmusmc.nl

PROVENANCE: Submitted article, peer reviewed.
Longitudinal biopsy studies, not only in children with problematic asthma but also in children with mild-to-moderate asthma, could shed some light on this, but ethical considerations will make such research extremely difficult.

Even more importantly, psychological issues, low socioeconomic class and nonadherence to treatment are widely recognised as factors making asthma difficult to treat. Although these problems may be recognised by physicians, specialised asthma nurses, home visits and electronic devices etc., these factors may be very hard to tackle in clinical practise. Improving adherence to treatment is probably the most cost-effective way of improving asthma treatment in children. eHealth may provide opportunities to monitor children more closely, improve adherence to treatment, increase self-management and, ultimately, improve asthma control. Many questions remain to be answered regarding e-health, the most important being: which children will benefit from e-health or telemonitoring?, how do you select them? and what should be monitored and how frequently? In particular, in children with problematic asthma e-health may facilitate more frequent and closer monitoring.

Little attention has been paid to children aged $<6$ yrs with problematic asthma. In this age group, different (inflammatory) pathophysiology and comorbidities, the role of viral infections and the lack of objective pulmonary function data may be even more challenging. Not all treatments, such as long-acting $\beta_{2}$-agonists (LABA) and omalizumab, are registered for use in preschool children and a separate work-up might be needed for these children.

Although children with problematic asthma claim time and high costs in a small group of patients, $95 \%$ of all asthmatic children have mild-to-moderate disease. HedLIN et al. [3] discuss interesting new ideas of asthma treatment in these children.

Preschool wheezers are a major problem to general practitioners and paediatricians. In 2008, a European Respiratory Society Task Force proposed the use of the phenotypes "episodic viral wheeze" and "multiple trigger wheeze"; suggesting that treatment decisions could be based on these phenotypes [6]. In everyday practice this division in subgroups does not seem very practical, and phenotypes change over time. For the physician faced with a preschool child with wheezing two questions are important. First, are there any atypical findings suggesting another diagnosis such as congenital heart disease, cystic fibrosis or ear, nose and throat pathology? Secondly, will this individual child benefit from anti-inflammatory treatment? Although much effort has been put in predicting a response to treatment in these preschool wheezing children, it still remains a challenge. 
The concept of "as needed" anti-inflammatory treatment in preschool wheezing children is attractive, given the fact that inhalation therapy in this age group is challenging, adherence to treatment in general is poor and overtreatment should be avoided. The intermittent use of high-dose nebulised budesonide in children with a positive "asthma predictive index" versus daily nebulised budesonide has been studied by ZEIGER et al. [7]. No differences in asthma exacerbations were found between the two groups. Although this study challenges the concept of maintenance anti-inflammatory treatment, the lack of a placebo group and the choice for nebulised budesonide with low lung deposition precludes the use of intermittent inhaled corticosteroids (ICS) in preschool children at the moment.

The concept of intermittent treatment with ICS was taken further by MARTINEZ et al. [8] in the TREXA (TReating Children to Prevent Exacerbations of Asthma) study, in which as needed inhaled steroids were compared with three other strategies in stable asthmatics with mild disease. However, this study clearly demonstrated that daily inhaled steroids are the most effective treatment in preventing asthma exacerbations. Rescue therapy with ICS during step-down in children with controlled asthma was more effective in preventing steroid courses than albuterol rescue treatment. However, one might argue that in children who had at least one course of prednisone during the past year, step-down therapy is not justified. The high percentage of steroid courses in these "well-controlled" children taking inhaled steroids urgently asks for phenotypes, clinical characteristics or biomarkers that might predict safe (intermittent) withdrawal of ICS in asthmatic children.

It is obvious that as asthma is a heterogeneous disease, there is no one size fits all approach. This was once again supported by the study of LEMANSKE et al. [9], which showed that the individual response to add-on therapy in children with uncontrolled asthma on low-medium doses of ICS is highly variable. In this study, predictors of response to treatment could not be defined except for a high Asthma Control Test score, which predicted a greater probability that the best response would be to step-up LABA.

Asthma guidelines focus highly on evidence-based conclusions of studies that show the average response to an intervention. However, in individual children, this response to treatment may vary greatly, including significant response, minimal response or even an adverse response. In addition, response to treatment may differ depending on the end-point that has been defined. An improvement in pulmonary function does not need to be accompanied by a reduction in symptoms or an improvement in quality of life. Therefore, there is a need for more personalised medicine, targeting the right patient with the right drug, with maximal effect and minimal side-effects.
Endotyping patients, defining subgroups of patients with certain genetic, clinical and biomarker profiles, opens the way to more personalised treatment and more personalised disease monitoring. In this respect, proteomic and metabolomic approaches increase expectations, although standardisation of techniques and methodological issues remain a challenge.

Comparative effectiveness research may be another novel option to learn more on individual responses to treatment in a daily life setting. With comparative effectiveness research, existing interventions are compared to determine which works best for which patients, and which poses the greatest benefits and the less harm in routine clinical practice. Access to electronic medical records is essential for this type of research, which may be additive to the traditional randomised controlled trials.

Targeting asthma with the right drug in the right patient to improve asthma control and asthma-related quality of life and reduce future risk should be our aim for the near future.

\section{STATEMENT OF INTEREST}

None declared.

\section{REFERENCES}

1 Bousquet J, Khaltaev N, eds. Global surveillance, prevention and control of chronic respiratory diseases: a comprehensive approach. Geneva, World Health Organization, 2007. www.who.int/gard/ publications/GARD\%20Book\%202007.pdf

2 Gustafsson PM, Watson L, Davis KJ, et al. Poor asthma control in children: evidence from epidemiological surveys and implications for clinical practice. Int J Clin Pract 2006; 60: 321-334.

3 Hedlin G, Konradsen J, Bush A. An update on paediatric asthma. Eur Respir Rev 2012; 21: 175-185.

4 Strunk RC, Weiss ST, Yates KP, et al. Mild to moderate asthma affects lung growth in children and adolescents. J Allergy Clin Immunol 2006; 118: 1040-1047.

5 Grol MH, Gerritsen J, Vonk JM, et al. Risk factors for growth and decline of lung function in asthmatic individuals up to age 42 years. A 30-year follow-up study. Am J Respir Crit Care Med 1999; 160: 1830-1837.

6 Brand PL, Baraldi E, Bisgaard H, et al. Definition, assessment and treatment of wheezing disorders in preschool children: an evidencebased approach. Eur Respir J 2008; 4: 1096-1110.

7 Zeiger RS, Mauger D, Bacharier LB, et al. Daily or intermittent budesonide in preschool children with recurrent wheezing. $N$ Engl J Med 2011; 365: 1990-2001.

8 Martinez FD, Chinchilli VM, Morgan WJ, et al. Use of beclomethasone dipropionate as rescue treatment for children with mild persistent asthma (TREXA): a randomised, double-blind, placebocontrolled trial. Lancet 2011; 377: 650-657.

9 Lemanske RF Jr, Mauger DT, Sorkness CA, et al. Step-up therapy for children with uncontrolled asthma receiving inhaled corticosteroids. N Engl J Med 2010; 362: 975-985. 